\title{
Contributions of Cell-extrinsic and Cell-intrinsic Factors to the Differentiation of a Neural-crest-derived Neuroendocrine Progenitor Cell
}

\author{
N. Mori,, S.J. BirRen, ${ }^{*}$ R. STEIN, $\ddagger$ D. STEMPle,${ }^{*}$ D.J. VANdenbergh, ${ }^{*}$ \\ C.W. WuENSCHELL, $\dagger$ AND D.J. ANDERSON $\dagger$ \\ *Division of Biology 216-76 and $\nmid$ Howard Hughes Medical Institute, California Institute of Technology, Pasadena, \\ California 91125; $\ddagger$ Department of Biochemistry, Tel Aviv University, Tel Aviv, Israel
}

A central question in developmental neurobiology concerns the mechanisms that generate cellular diversity in the vertebrate nervous system. Cell lineage analyses have established that many progenitor cells in the developing nervous system are multipotent (Turner and Cepko 1987; Holt et al. 1988; Wetts and Fraser 1988). However, the mechanisms that control the differentiation of such progenitor cells are poorly understood. It is assumed that both the developmental history of a progenitor cell and its interactions with its local environment influence its final choice of developmental fate. Few experimental systems are available, however, in which it has been possible to dissect the relative contributions of these two factors to the differentiation program. In the vertebrate nervous system, only two well-defined multipotential progenitor cells have been isolated and studied in detail. These are the O2A progenitor, which gives rise to two types of glial cells in the central nervous system (Raff 1989), and the sympathoadrenal progenitor (Doupe et al. 1985b; Anderson and Axel 1986), which generates either the sympathetic neurons or adrenal medullary chromaffin cells of the peripheral nervous system.

The Sympathoadrenal Progenitor Has a Restricted Repertoire of Developmental Fates

Sympathoadrenal progenitors derive from a population of neural crest cells that detach from the top of the neural tube and migrate throughout the embryo. Lineage tracing studies, performed both in vitro (Sieber-Blum and Cohen 1980; Baroffio et al. 1988) and in vivo (Bronner-Fraser and Fraser 1989), have indicated that single cells in the premigratory crest are initially multipotent and are able to generate most or all crest derivatives. These include not only sympathetic neurons and chromaffin cells, but other neuronal populations such as sensory and parasympathetic, as well as nonneuronal cell types including glia and melanocytes. As neural crest cells migrate and localize in the periphery, however, they appear to become developmentally restricted, perhaps analogous to the gradual narrowing of the developmental potential observed in hematopoiesis (Anderson 1989). The sympathoadrenal progenitor appears to represent one such developmentally restricted progenitor cell. These progenitors are first detectable concomitant with the aggregation of crest cells to form the sympathetic ganglion primordia (Fig. 1). They can be visualized using several antigenic markers including tyrosine hydroxylase (TH), the ratelimiting enzyme in catecholamine biosynthesis (Cochard et al. 1979). After this initial aggregation, some of the cells in this population remain in the ganglion primordia and differentiate into sympathetic neurons, whereas others continue migrating ventrally to invade the mesodermally derived adrenal primordium (Fig. 1). The latter proliferate and differentiate into chromaffin cells, which ultimately form the medullary zone of the adrenal gland.

Experiments in primary cell culture originally suggested that environmental factors determine the ultimate fate of progenitors in this sublineage. It was shown that postnatal (Unsicker et al. 1978) or even adult (Doupe

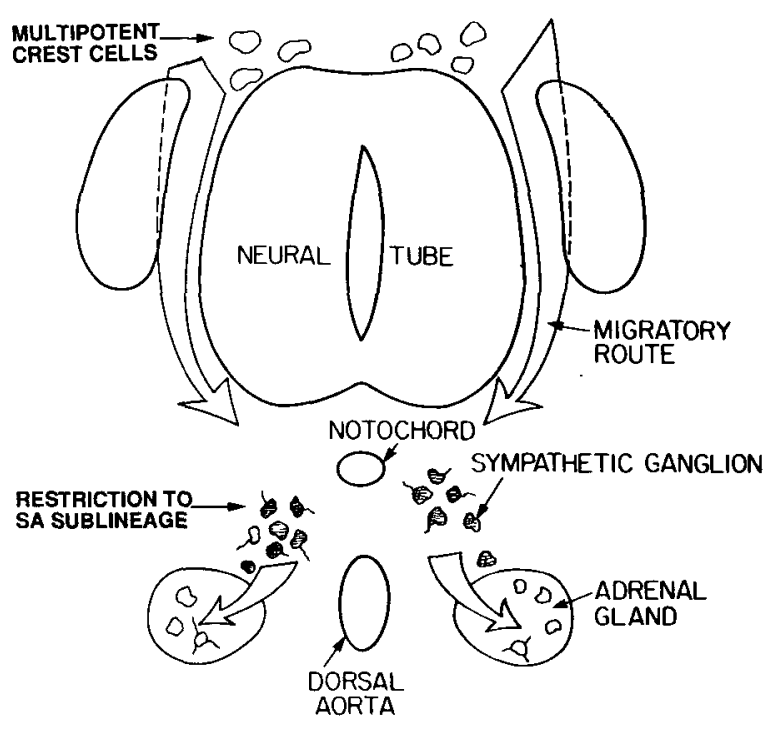

Figure 1. Schematic indicating the migratory route taken by cells in the sympathoadrenal lineage. A cross section through the spinal cord in the thoraco-lumbar region is shown. 
et al. 1985a) chromaffin cells could be induced to transdifferentiate into sympathetic neuron-like cells by nerve growth factor (NGF). This transdifferentiation could be slowed or blocked by glucocorticoid (GC), suggesting that the high local concentration of these steroids in the surrounding adrenal cortex could similarly inhibit the neuronal differentiation of chromaffin precursors in vivo (Unsicker et al. 1978). The idea that the microenvironment of sympathoadrenal lineage cells is important in controlling their differentiation in vivo is supported by the observation that $\mathrm{TH}^{+}$cells in the sympathetic ganglion and the adrenal gland acquire distinct antigenic phenotypes only a few days after migration has ended (Fig. 2).

To determine whether individual sympathoadrenal progenitors are bipotential, we have isolated these cells from fetal rat adrenal glands, using cell-surface monoclonal antibodies and fluorescence-activated cell sorting

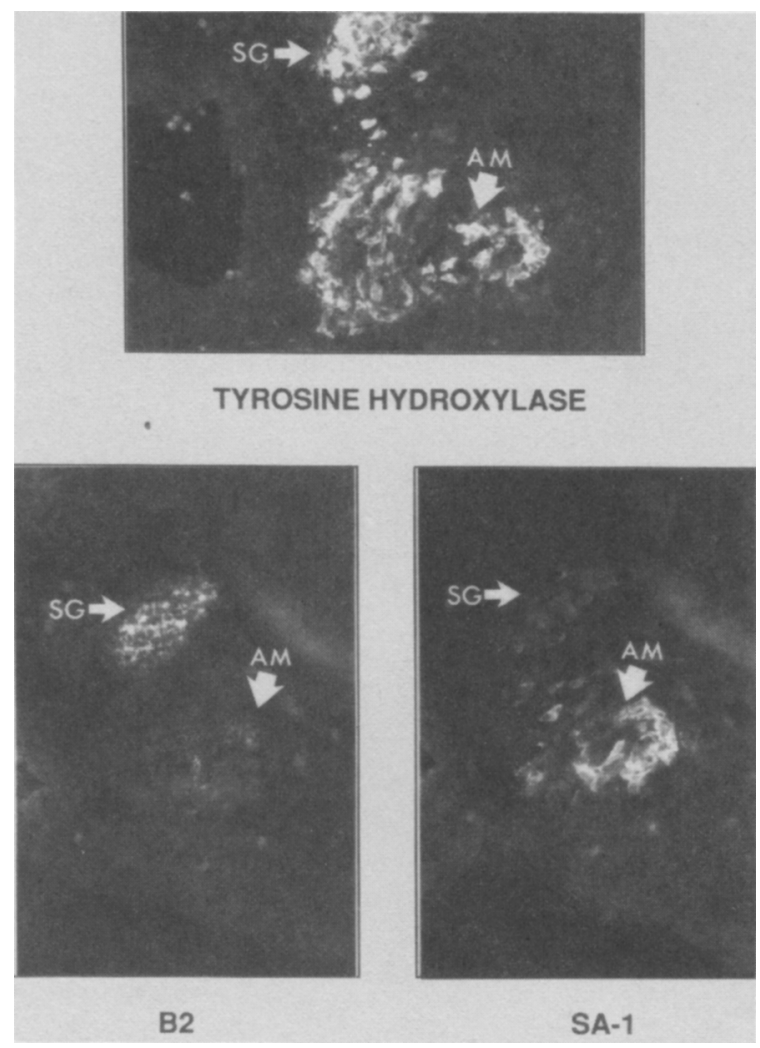

Figure 2. Differential expression of antigenic markers by embryonic sympathoadrenal cells in the ganglion and adrenal primordia in vivo. Shown are sections through the caudal thoracic region of an E15.5 rat embryo. Cells in both the sympathetic ganglion (SG) and adrenal medulla (AM) stain with an antibody to TH (top). Note the individual cells between the SG and AM, which may be migrating to the adrenal. (Bottom) Fluorescence photomicrographs of the same section double-labeled with monoclonal antibody B2 (left). which selectively labels primitive sympathetic neurons (Anderson and Axel 1986) and SA-1 (right), which selectively labels adrenal chromaffin cells. (J. Carnahan and P.H. Patterson, unpubl.).

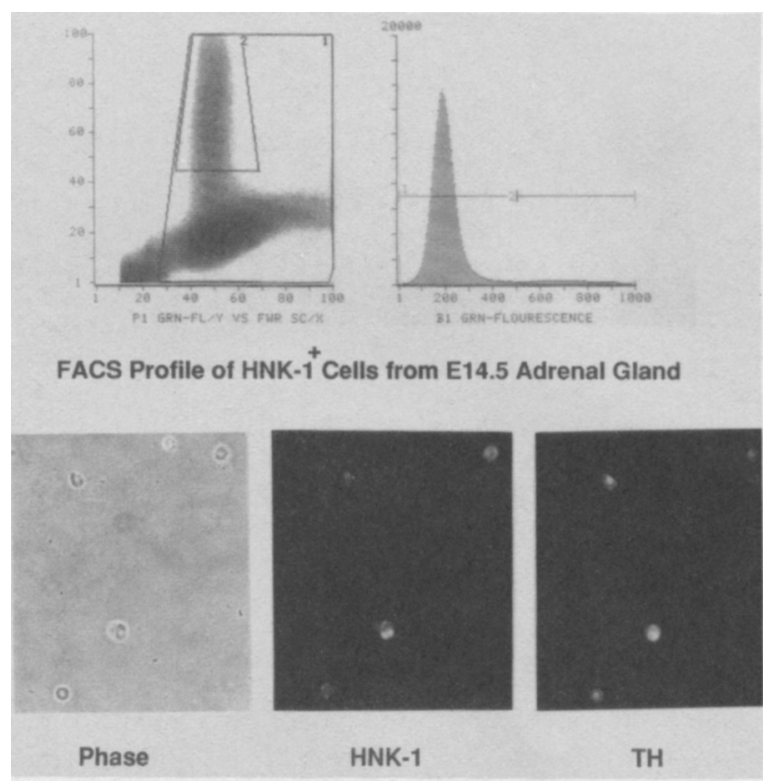

Figure 3. Isolation of sympathoadrenal progenitor cells from E14.5 rat adrenal gland by FACS using monoclonal antibody HNK-1. (Top, left) Forward light scatter (abscissa) versus fluorescence intensity (ordinate). Gated region 2 delimits cell population isolated. (Top, right) Histogram of fluorescence intensity versus cell number. The $\mathrm{HNK}-1^{+}$cells represent approximately $10 \%$ of the adrenal population. (Bottom) FACS-isolated progenitors several hours after plating. The same field is shown photographed with phase contrast optics (left), fluorescein-HNK-1 (middle), or rhodamine-TH (right). Note that all the $\mathrm{HNK}-1^{+}$cells are also $\mathrm{TH}^{+}$.

(FACS) (Anderson and Axel 1986; Anderson 1988) (Fig. 3). In culture, the differentiation of such progenitors into chromaffin cells (Fig. 4) is absolutely dependent on the addition of exogenous GC to the culture medium (Anderson and Axel 1986; Seidl and Unsicker 1989; A. Michelsohn and D.J. Anderson, unpubl.). In the absence of GC, these progenitors initiate neuronal differentiation (Fig. 4), a process that can be promoted by specific growth factors (see below). Quantitative analysis of cell populations and serial observations of single cells strongly suggest that individual progenitors in these cultures are indeed bipotential (Anderson and Axe 1986). Similar observations have been made for progenitors isolated from postnatal (Doupe et al. 1985b) and, more recentiy, fetal superior cervical sympathetic ganglia (J. Carnahan and P.H. Patterson, pers. comm.). This conclusion is supported by recent in vivo observations showing that, in the ganglion primordia, chromaffin-specific and neuronspecific markers are transiently coexpressed by individual progenitor cells (D.J. Anderson et al., unpubl.). Taken together, these data suggest that a common, developmentally restricted progenitor cell initially populates both the sympathetic ganglia and the adrenal gland and then differentiates into either sympathetic neurons or chromaffin cells under the influence of factors differentially distributed in these alternative sites of migratory arrest. 


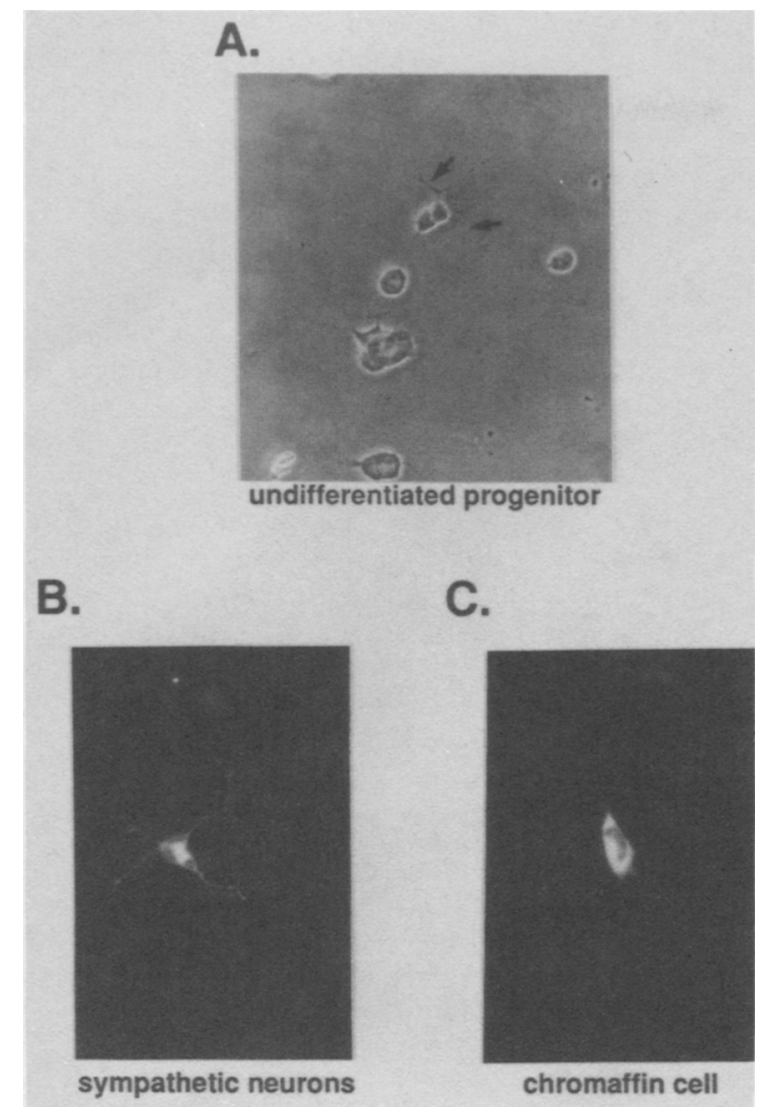

Figure 4. FACS-isolated progenitors develop into sympathetic neurons or chromaffin cells, in vitro. $(A)$ Phasecontrast micrograph of progenitor cells after 24 hours in culture. $(\rightarrow)$ Note the short cytoplasmic extensions characteristic of such cells. $(B)$ Progenitors after 2-3 days in serum-containing medium. Under these conditions, the cells begin to differentiate into neurons, extending short neurites, but they eventually die. Neurite extension is enhanced by addition of FGF (not shown). (C) Progenitors after 2-3 days in $5 \mu \mathrm{M}$ dexamethasone. Note the difference in morphology from the cells in $B$. Cells in both $B$ and $C$ are labeled with an anti-TH antibody. However, cells such as those in $B$ also express neurofilaments, whereas those in $C$ express the chromaffin marker PNMT (not shown).

\section{Neuronal Differentiation of Sympathoadrenal Progenitors May Be Controlled by Polypeptide Factors Acting in a Cascade}

The requirement of GC for chromaffin cell differentiation in vitro is consistent with the fact that in vivo these cells normally develop within the adrenal gland, the major site of GC synthesis. In contrast, the identification of factors promoting neuronal differentiation and their relationship to molecules present in vivo has been more difficult to establish. As NGF converts both chromaffin cells (Unsicker et al. 1978) and PC12 cells (Greene and Tischler 1976) into sympathetic neuronlike cells, this molecule initially seemed like a reasonable candidate for the factor that promotes neuronal differentiation of sympathetic neuroblasts in vivo. However, subsequent studies of the timing and location of NGF synthesis in the embryo indicated that NGF is unlikely to be playing such a role. NGF is initially produced in the targets of sympathetic innervation, not in the ganglia, and is not made until the neurons have already grown their axons to these targets (Davies et al. 1987; Korsching and Thoenen 1988). Moreover, freshly isolated sympathoadrenal progenitor cells are initially unresponsive to NGF in vitro (Anderson and Axel 1986; Ernsberger et al. 1989). Such findings have led to the search for other factors that might promote neuronal differentiation in this system.

One such factor may be fibroblast growth factor (FGF). FGF is the only factor other than NGF able to induce the expression of neuron-specific mRNAs in PC12 cells (Leonard et al. 1987; Stein et al. 1988a). Moreover, both basic (Stemple et al. 1988) and acidic (Claude et al. 1988) FGF can promote the transdifferentiation of postnatal chromaffin cells into neurons. However, FGF cannot act subsequently as a survival factor for the sympathetic neurons generated from chromaffin cells. Rather, FGF induces an NGF dependence in these cells (Stemple et al. 1988). Taken to-

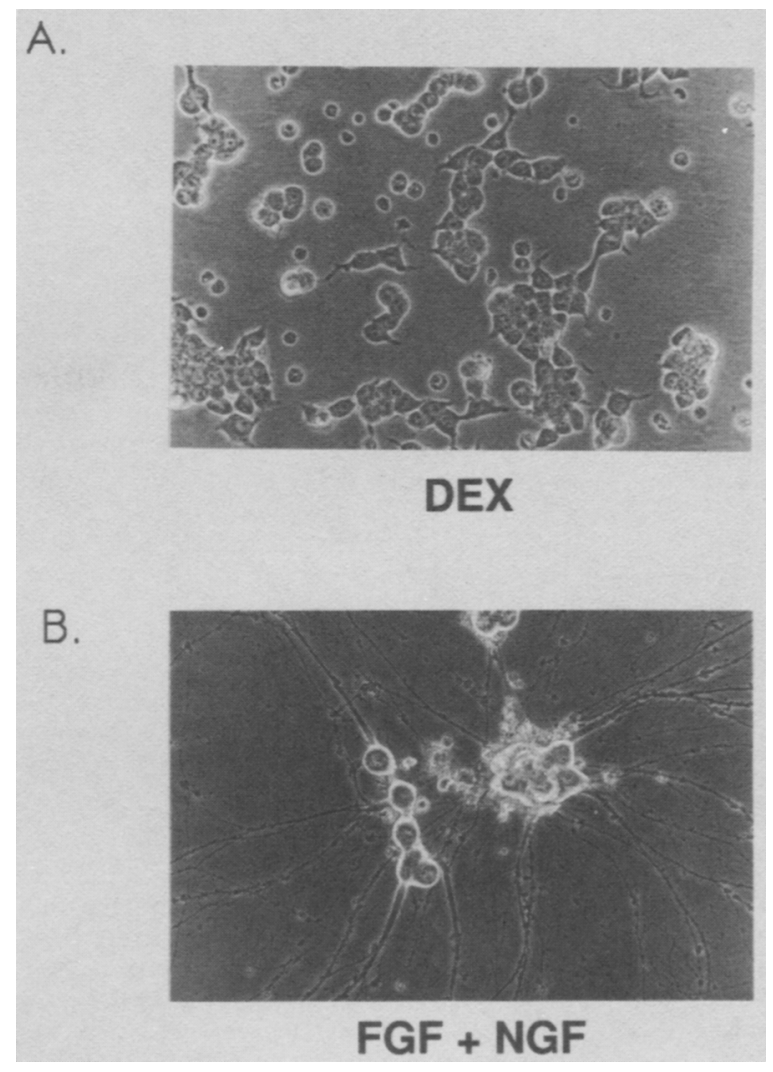

Figure 5. Immortalized sympathoadrenal progenitor cells can differentiate into stable, postmitotic neurons. $(A) \mathrm{MAH}$ cells maintained in $5 \mu \mathrm{M}$ dexamethasone. Note the morphologic similarity to the primary cells shown in Fig. 4A. $(B)$ A small proportion $(<0.1 \%)$ of $\mathrm{MAH}$ cells develop into neurons in the presence of bFGF $(10 \mathrm{ng} / \mathrm{ml})+\mathrm{NGF}(50 \mathrm{ng} / \mathrm{ml})$. The cells shown had been maintained for approximately 4 weeks in culture at the time this photograph was taken. The magnification in $A$ is twice that in $B$. 
gether, these results suggested a potential role for FGF in the initial stages of neuronal differentiation in the sympathoadrenal lineage.

To address this question, we first generated immortal cell lines from sympathoadrenal progenitors by retroviral transduction of the v-myc oncogene (Fig. 5A). These lines appear to have retained many of the morphologic and antigenic properties of their normal (i.e., nonimmortalized) counterparts (Birren and Anderson 1990). Such cell lines facilitate cell biological and molecular experiments by providing large numbers of easily available cells. We have named these cell lines MAH cells, for myc-infected, adrenal-derived. $H \mathrm{NK}$ 1-positive cells (Birren and Anderson 1990). MAH cells represent the first case where a well-defined neuronal progenitor population has been first isolated and then immortalized.

A striking feature of $\mathrm{MAH}$ cells is that they respond to basic FGF (bFGF) by extending long neurites, but they do not do so in response to NGF. This is because these cells do not express the NGF receptor (NGFR), as determined by hybridization of $\mathrm{MAH}$ cell mRNA with cloned NGFR cDNA probes (Birren and Anderson 1990). Like normal chromaffin cells, MAH cells

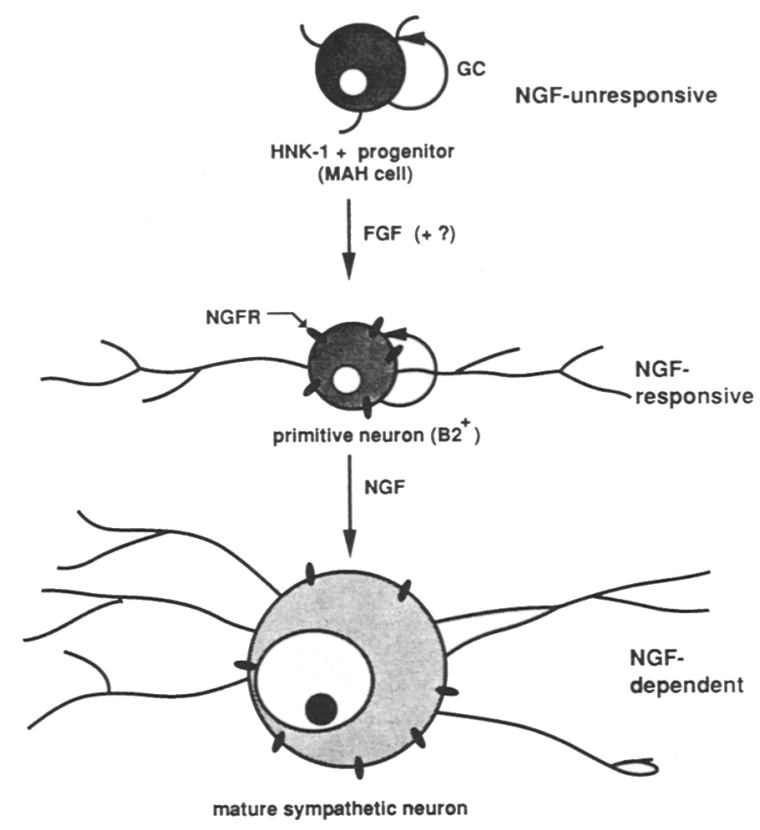

Figure 6. Stages of neuronal differentiation in the sympathoadrenal lineage. The sympathoadrenal progenitors represented by MAH cells (top) extend neurites in response to FGF but do not yet express NGF receptors. In the presence of glucocorticoid, these cells divide (circular arrow) but do not differentiate. Following treatment with FGF, a primitive neuron can be observed that bears processes and is still dividing. This cell type has been described in primary cultures previously (Anderson and Axel 1986; Anderson 1988). At least a subpopulation of these primitive neurons begins to express the NGF receptor. In the presence of NGF, these cells mature into postmitotic neurons with large cell bodies, prominent nucleoli, and extensive networks of neurites. Such neurons are then dependent on NGF for survival. (Reprinted, with permission, from Birren and Anderson 1990.) induced to differentiate with FGF alone die several days after extending neurites. In the presence of both FGF and NGF, however, a small proportion of MAH cells become postmitotic neurons that are stable for many weeks in culture if supplied with NGF (Fig. 5B). In the presence of a blocking anti-NGF antibody, however, these $\mathrm{MAH}$-derived neurons rapidly die, indicating that they are now dependent on the neurotrophic factor for survival. Northern blot analysis has confirmed that treatment of MAH cells with FGF induces the de novo expression of NGFR mRNA (Birren and Anderson 1990).

Taken together, these data suggest that neuronal development in this lineage may involve a relay, or cascade, in which one factor (perhaps FGF) initiates differentiation and simultaneously primes the cell to respond to a second factor (NGF), which then subserves further maturation and survival (Fig. 6). The extent to which FGF exerts such an effect on normal progenitors is now under investigation. Interestingly, when cultured at high density, such progenitors appear to initiate neuronal differentiation spontaneously. Such an effect could be due to an autocrine action of FGF or an FGF-like molecule. Whatever the case, the data suggest that the initial stages of neuronal differentiation in this lineage occur independently of NGF (Coughlin and Collins 1985) but involve an induction of the NGFR. As NGF appears able to up-regulate expres-

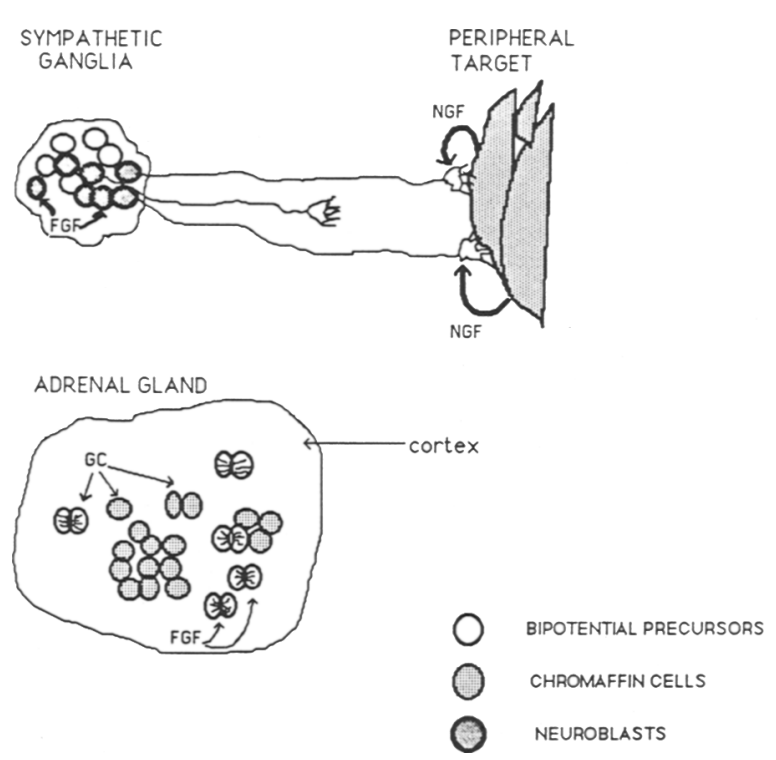

Figure 7. A two-stage model for sympathetic neuron development. (Top) FGF (or a different factor with similar biological activity) induces progenitor proliferation and neuronal differentiation locally in the sympathetic ganglion primordia. These differentiating cells extend processes toward their targets. The appearance of NGF in targets coincides with the arrival of the sympathetic afferent fibers. At or near the target, neurons acquire responsiveness to and dependence on NGF, which thereby supports their survival and further maturation. (Bottom) In the adrenal gland, GC permits FGF to mitotically expand the developing endocrine population but prevents the factor from inducing neuronal differentiation. 
sion of its own receptor (at least in PC12 cells; Doherty et al. 1988), it may be that the initiating factor simply provides sufficient NGFR to establish an NGF autoregulatory loop that is in turn primarily responsible for the induction of full NGF-responsiveness and NGFdependence.

This "two-factor" model would fit well with the fact that, in vivo, neuronal differentiation is initiated at a site (the ganglia) distant from that where NGF is eventually synthesized (Korsching and Thoenen 1988) (Fig. 7). According to this view, FGF (or a molecule with similar biological activity) would act locally in the ganglia to trigger initial neurite outgrowth. At the same time, the induction of NGFR expression would ensure that by the time the growing axons arrived at the periphery, they would have acquired competence to be supported by target-derived NGF. Moreover, the induction of an absolute NGF dependence in these growing neurons would ensure the death of any cells that failed to grow to an appropriate target. Studies are now in progress to determine whether mRNAs encoding FGF or related members of the FGF gene family (Hébert et al. 1990) are indeed present in embryonic sympathetic ganglia at the stage when neuronal differentiation normally occurs. Recent immunocyto- chemical evidence suggests that bFGF, at least, is present in embryonic chick sympathetic neurons (Kalcheim and Neufeld 1990).

\section{Derepression of Neural-specific Genes May Be a Developmental Prerequisite for Their Regulation by Environmental Factors}

The foregoing data indicate that the development of sympathoadrenal derivatives occurs through the action of diffusible factors on a progenitor cell with a restricted repertoire of developmental fates. Studies of gene expression in $\mathrm{PC} 12$ cells indicate that, at the molecular level, these factors act by up- and downregulating terminal-differentiation genes specific to the neuronal and chromaffin phenotypes, respectively (Leonard et al. 1987; Stein et al. 1988a). In other words, NGF and FGF induce neuron-specific genes and suppress chromaffin-specific genes, and vice versa for GC. The "repertoire of fates" in a committed sympathoadrenal progenitor cell is therefore reflected molecularly in batteries of neuron-specific and chromaffin-specific genes that can be activated or repressed by FGF/NGF and GC. The question thus is how this repertoire first becomes established in the pro-

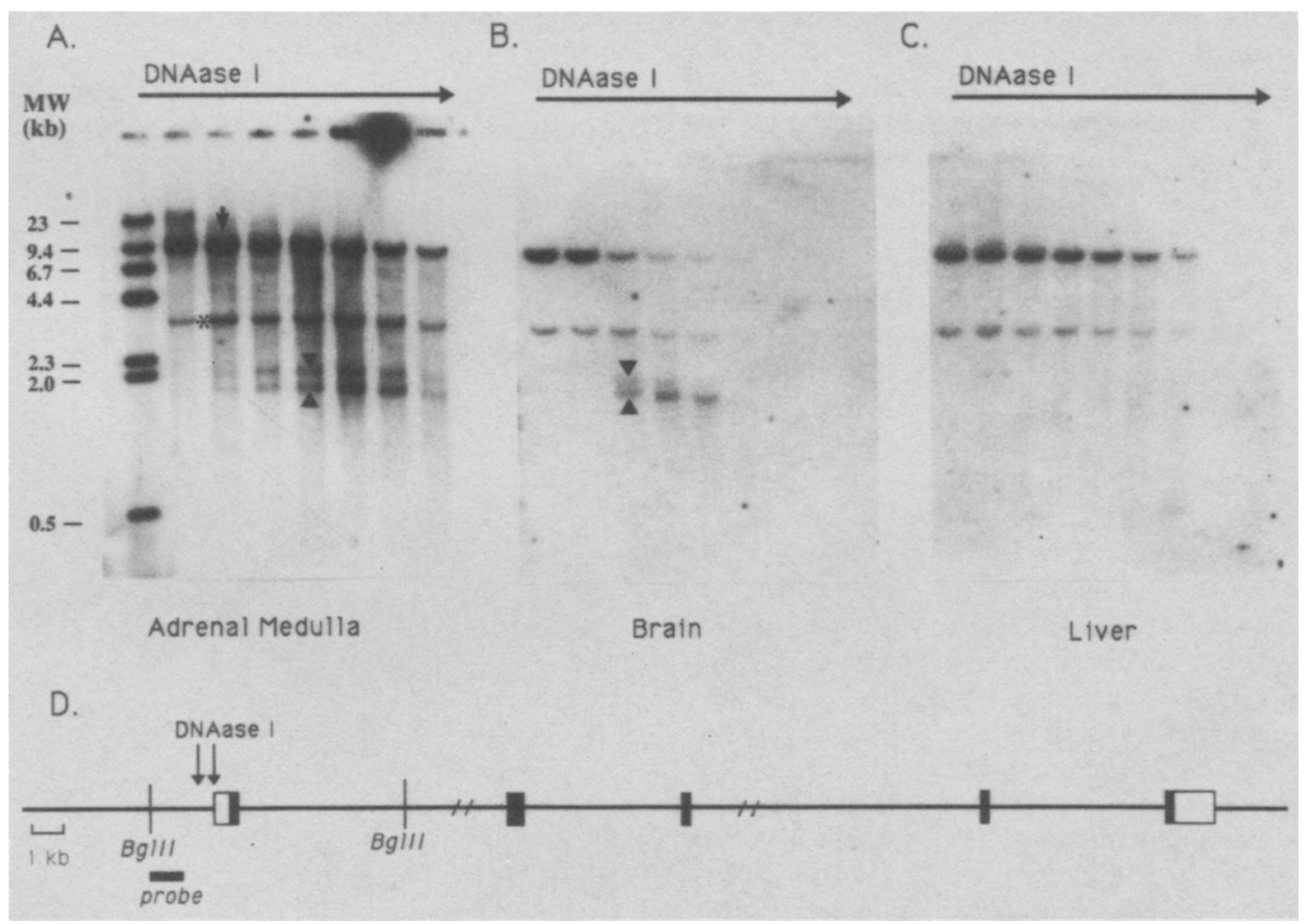

Figure 8. Identification of two DNase $1 \mathrm{HSS}$ in the SCG10 gene that are lineage-specific. $(A, B, C)$ Southern blots of DNase-1-digested, $B g l$ II-restricted genomic DNA from adult AM $(A)$, E18 brain $(B)$, and liver $(C)$. The two HSS are indicated by the presence of the two subbands running at 1.8 and $2 \mathrm{~kb}(A$ and $B$, arrowheads). The arrow pointing down indicates the $8-\mathrm{kb}$ $B g l \mathrm{II}$ parent band $(A)$. The faint band at $\sim 3 \mathrm{~kb}$ (asterisk) represents cross-hybridization of the probe to the immediately

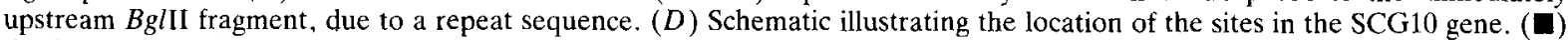
Coding exons; ( $\square$ ) noncoding exons. The complete intron-exon organization of this gene has not been determined. Vertical lines demarcate the 8-kb BglII parent band containing the HSS. The size and location of the probe are indicated. (Reprinted, with permission, from Vandenbergh et al. 1989.) 
CONSTRUCT

STRUCTURE

CAT37

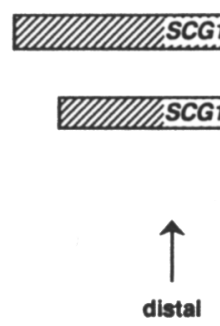

САT30

CAT4

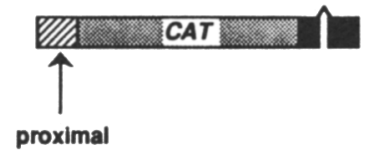

KB OF SCG10 5' DNA

3.5

0.55
NO. OF MICE

4

3

Figure 9. Schematic illustrating SCG10-CAT gene fusion constructs used to generate transgenic mice. The "proximal" region in CAT4 extends from a $P v u l l$ site in the $5^{\prime}$-untranslated region of the mRNA to an EcoRI site $\sim 0.5 \mathrm{~kb}$ upstream. The distal region in CAT37 contains an additional $3.7 \mathrm{~kb}$ of $5^{\prime}$-flanking DNA. CAT30 is $0.7 \mathrm{~kb}$ shorter than CAT37 at its $5^{\prime}$ end. The number of independent integrants generated for each construct is indicated. For each integrant, between 1 and 30 copies of the gene were present. (Reprinted, with permission, from Wuenschell et al. 1990.)

genitor cell. The determination of this repertoire is presumably closely related to the commitment of early neural crest cells to the sympathoadrenal sublineage.

Both FGF and GC are factors that exert a broad range of effects on cells of different lineages through the activation of transcription factors. In the case of $\mathrm{GC}$, the receptor itself is a transcription factor (Chandler et al. 1983); in the case of FGF (and NGF), the receptors activate transcription factors indirectly via second messenger pathways (Sheng and Greenberg 1990). The specificity of action of these factors on cells of a given lineage must therefore be determined, at least in part, by the set of target genes that their corresponding transcription factors can activate or repress. One mechanism that has been proposed to account for the selectivity of target gene activation is the accessibility of such genes to transcription factors in chromatin (Robins et al. 1982; Burch and Weintraub 1983). According to this view, the repertoire of fates available to a sympathoadrenal progenitor cell would be established as a set of genes maintained in a chromatin configuration accessible to transcription factors activated by FGF and/or GC (Anderson and Axel 1985).

As an initial test of this hypothesis, we examined the chromatin structure of a neuron-specific gene that can be induced by FGF or NGF in sympathoadrenal cells. This gene, SCG10, encodes a $22-\mathrm{kD}$ vesicle-associated protein that accumulates in the growth cones of developing and regenerating neurons (Stein et al. 1988b). We examined SCG10 chromatin structure in adult adrenal chromaffin cells because these cells retain the capacity to transdifferentiate into neurons in response to FGF or NGF, and because SCG10 is induced during this transdifferentiation (Anderson and Axel 1985). As an index of an accessible or "open" chromatin structure, we used the well-established criterion of sensitivity to digestion by DNase I (Gross and Garrard 1988).

We found that SCG10 exhibits a specific pattern of DNase I hypersensitivity in the nuclei of adult chromaffin cells (Vandenbergh et al. 1989). This pattern, marked by two cleavage sites in the promoter region of the gene (Fig. 8A), is also found in neurons expressing high levels of SCG10 (Fig. 8B) but not in nonneuronal cells (Fig. 8C). Thus, these hypersensitive sites appear to define a state of the SCG10 gene that is characteristic of both neurons and chromaffin cells and is therefore independent of whether or not the gene is expressed at high levels. We anticipate but have not yet proven that these sites appear in ontogeny at the time that neural crest cells initially commit to the sympathoadrenal lineage, since this is the earliest time that the SCG10 gene is detectably transcribed (Anderson and Axel 1986). The persistence of this DNase-sensitive chromatin structure in adult chromaffin cells, moreover, could in part account for the phenotypic plasticity exhibited by these cells (Vandenbergh et al. 1989).

To gain further insight into the mechanisms controlling the assembly of this state of the SCG10 gene, we

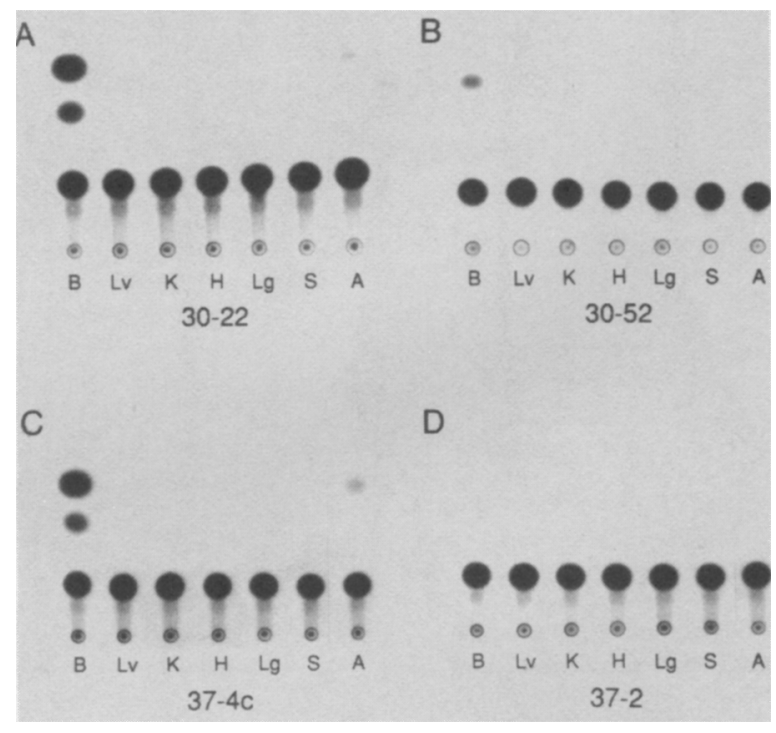

Figure 10. Neuron-specific expression of CAT37 and CAT30 in transgenic mice. Two representative integrants for CAT30 $(A, B)$ and CAT37 $(C, D)$ are shown. CAT enzymatic activity was assayed using $50 \mu \mathrm{g}$ of protein from the indicated adult tissues: (B) brain; (Lv) liver; (K) kidney; (H) heart; (Lg) lung; (S) spleen; (A) adrenal gland. $(A, B) 40 \mu \mathrm{g}$ of adrenal gland protein was used. Note that in the two lines expressing the highest levels of CAT in brain $(A, C)$ a low level of expression is also detected in the adrenal gland. (Reprinted, with permission, from Wuenschell et al. 1990.) 
have examined the formation of the hypersensitive sites in transgenic mice (Wuenschell et al. 1990). We generated several mice containing varying lengths of SCG10 5 -flanking DNA fused to the heterologous reporter gene CAT (bacterial chloramphenicol acetyltransferase) (Fig. 9). All of these gene fusion constructs contained the proximal region of the SCG10 promoter to which the DNase-I-hypersensitive sites map in the endogenous gene. However, these constructs varied in the amount of SCG10 DNA lying upstream of this region (Fig. 9).

We found that $3.5 \mathrm{~kb}$ of SCG10 5 -flanking DNA appeared sufficient to reconstitute correctly the pattern

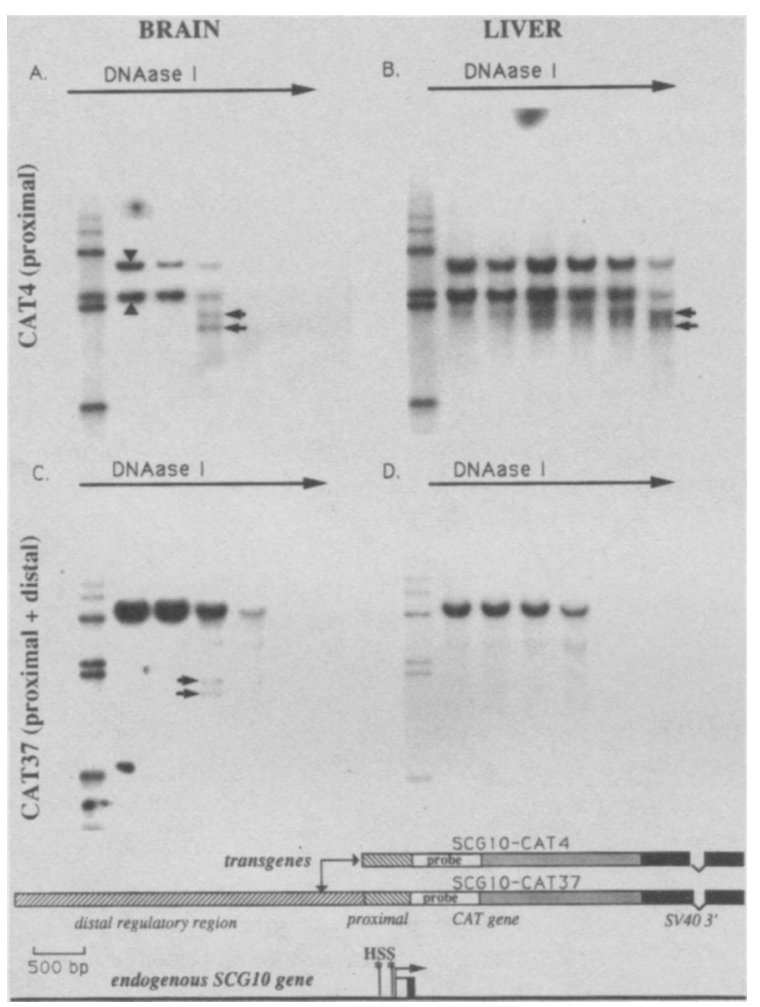

Figure 11. Formation of the HSS in transgenic mice containing SCG10-CAT gene fusion constructs. The HSS were assayed in the brains $(A, C)$ and livers $(B, D)$ of two transgenic mice expressing either the transgene SCG10-CAT4 $(A, B)$ or SCG10-CAT37 $(C, D)$. The structure of these two gene fusion constructs relative to the endogenous SCG10 gene is illustrated below the Southern blots. Note that the twin HSS map within the proximal regulatory region contained within both transgene constructs. CAT4 $(A, B)$, which contains only the 450-bp proximal regulatory region, contains the HSS in both tissues ( $A, B$, horizontal arrows). CAT37 $(C, D)$, which contains the entire 4-kb 5 region of SCG10, is expressed in brain nuclei but not liver nuclei and contains the HSS only in brain nuclei ( $C$, horizontal arrows). The band at $\sim 3 \mathrm{~kb}$ reflects a weak HSS that is also present in the endogenous gene but is not tissue-specific (data not shown). The probe used (schematic) contains only CAT coding DNA and therefore detects only the transgene on these Southern blots. Two parent bands are detected in the CAT4 mouse $(A, B$, arrowheads) due to the tandem ligation of two copies of the transgene. A single band is detected for the CAT37 mouse. (Reprinted, with permission, from Vandenbergh et al. 1990.) of endogenous SCG10 gene expression, at least at the gross tissue level (Wuenschell et al. 1990). A high level of transcription was detected in brain tissue, a very low level in adrenal gland tissue, and no expression in various nonneuronal tissues (Fig. 10). Importantly, in these mice, the two DNase-hypersensitive sites formed in their characteristic position in the transgene in a neuron-specific manner (Fig. 11C,D) (Vandenbergh et al. 1989). Surprisingly, deletion of the distal region of the SCG10 5'-flanking DNA (Fig. 9, CAT4) resulted in deregulated expression of the transgene (Fig. 12A-C). In these mice, moreover, the hypersensitive sites formed in their characteristic position in liver, an inappropriate tissue, as well as in brain tissue (Fig. 11A,B).

These results suggested that the two hypersensitive sites reflect a state of the SCG10 gene that can, in principle, form in any cell type. The formation of this state is apparently blocked in nonneuronal cells by sequences lying upstream of the proximal hypersensitive region. In an independent set of transient expression experiments (Mori et al. 1990), we showed that this upstream region has the properties of a silencer, which strongly suppresses transcription from the SCG10 promoter in nonneuronal, but not in neuronalcell lines (Fig. 13). This silencer, moreover, can act in an orientation-independent and relatively positioninsensitive manner to suppress transcription from a heterologous promoter as well (Fig. 14).

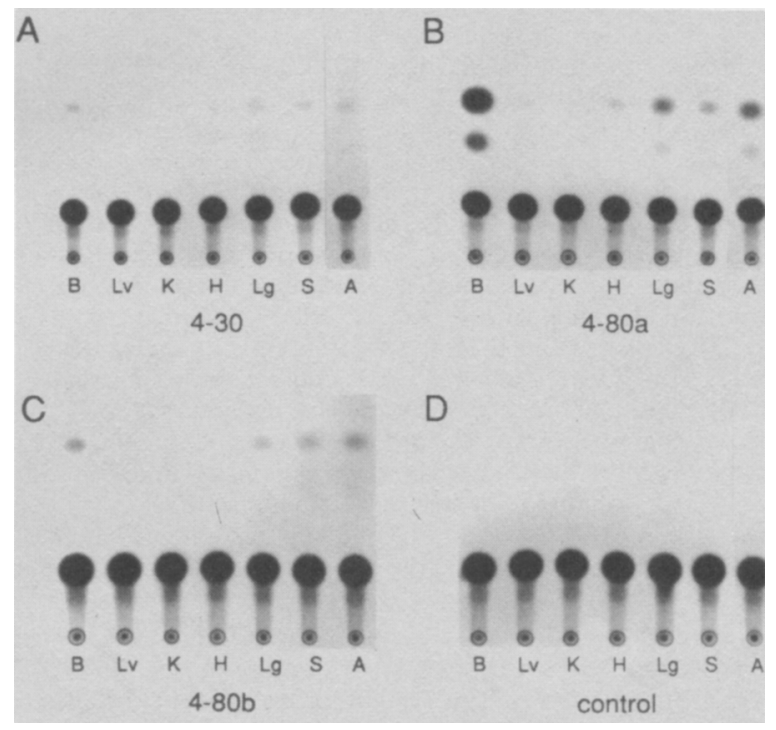

Figure 12. SCG10-CAT constructs lacking the distal silencer region exhibit deregulated expression in transgenic mice. Shown are CAT assays from three different integrants harboring the CAT4 construct in $A, B$, and $C$, respectively. In $D$, a nontransgenic control is shown. Tissue abbreviations are the same as those in Fig. 10. Note that varying levels of CAT activity can be detected in some or all nonneuronal tissues in all three transgenic mice. Quantitative analysis of such assays indicates that despite their loss of tight tissue specificity, the relative level of CAT activity is always highest in brain tissue for each mouse (Wuenschell et al. 1990). 


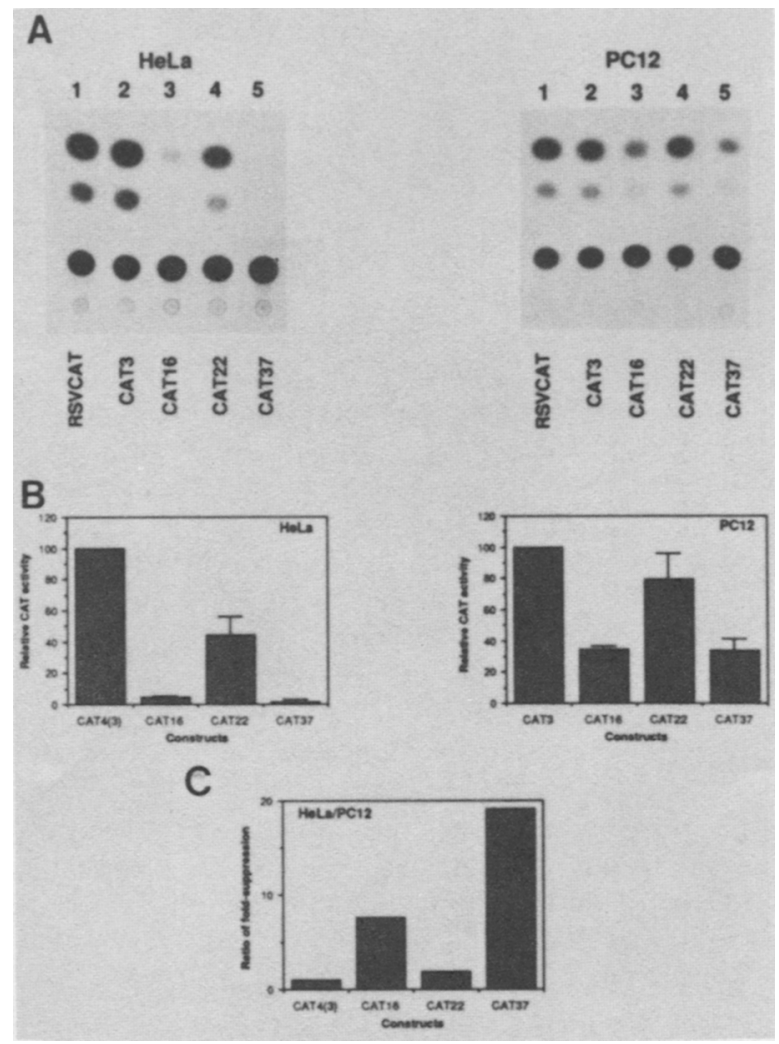

Figure 13. Identification of a constitutive enhancer in the proximal region and of a cell-preferred silencer in the distal region. (A) Representative CAT enzymatic activity assays from an experiment in which the indicated constructs were transfected into HeLa and PC12 cells, in parallel. CAT3 contains the promoter-proximal region and is similar to CAT4 (Fig. 9). Note that the activity of CAT3 is comparable to that of RSV-CAT in both HeLa and PC12 cells, and that CAT37 and CAT16 are strongly suppressed in HeLa cells relative to CAT3. (B) Quantification of the suppression activity. CAT activity was normalized to that of the cotransfected internal control plasmid, pRSVlacZ. The activity of CAT3 (or CAT4) was set at $100 \%$, and the fractional activity shown by the other constructs was calculated. The data shown are the mean \pm range of two independent experiments. (C) Suppression by CAT37 and CAT16 is stronger in HeLa cells than in PC12 cells. Fold suppression was calculated for each construct in each cell line as the reciprocal of the activity relative to CAT3 (from $B$ above). The ratio of this value for HeLa and PC12 cells was then calculated. Thus, for example, CAT37 is suppressed 55-fold in HeLa cells and 2.8-fold in PC12 cells (relative to CAT3), so the ratio of fold suppression is 19.4. (Reprinted, with permission, from Mori et al. 1990.)

Since deletion of the silencer permits an SCG10 transgene to assume an accessible chromatin configuration in an inappropriate tissue, it is reasonable to conclude that the endogenous gene acquires this configuration during normal development because of the loss or inactivation of proteins that interact with the silencer. Thus, the open state of the SCG10 gene in sympathoadrenal progenitors may reflect the fact that this gene has undergone a specific derepression event (Fig. 15). It is not clear whether this derepression occurs by an actual "opening" of chromatin or simply by the relief

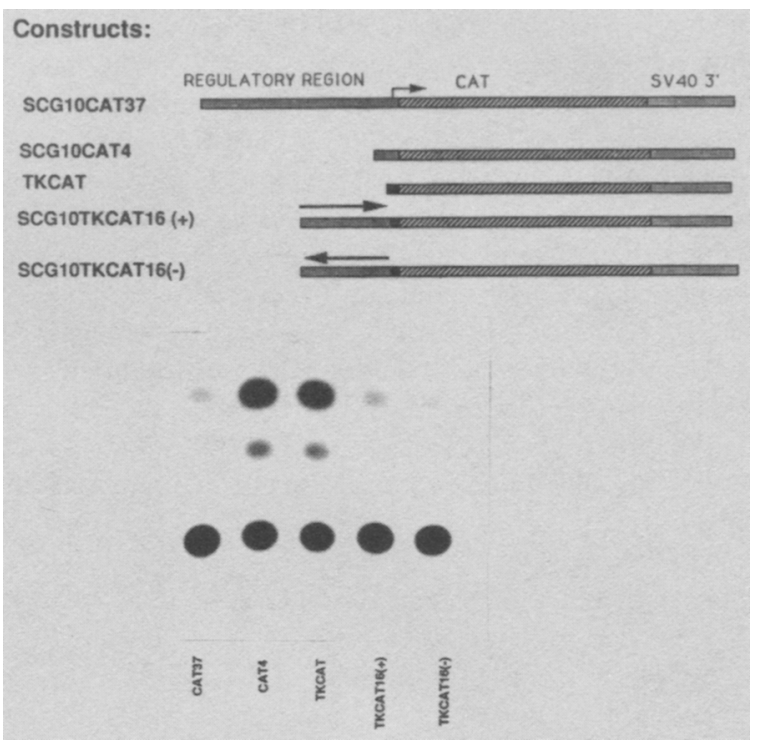

Figure 14. Orientation-independent inhibition of transcription from a heterologous promoter by the SCG10 silencer element. The constructs shown were assayed by transient transfection in HeLa cells. TKCAT contains the HSV TK promoter to position -109 (McKnight and Kingsbury 1982). SCG10CAT 16(t) contains $1.6 \mathrm{~kb}$ of SCG10 DNA located immediately upstream of the proximal promoter-enhancer region contained in CAT4 (for details, see Mori et al. 1990). Note that in either orientation, this DNA suppresses transcription from the TK promoter.

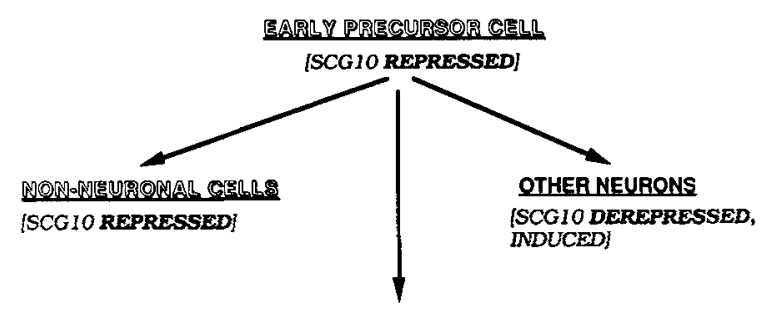

SYMPATHOADRENAL PROGENITOR [SCGIO DERREPRESSED, UNINDUCED]

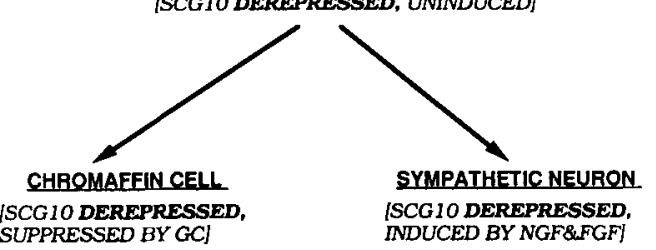

Figure 15. Model to suggest the role of the silencer element during neuronal lineage diversification. Early neuroepithelial precursor cells maintain the $S C G 10$ gene in a repressed state. With the appearance of committed sympathoadrenal progenitor cells, the gene becomes derepressed as a result of functional inactivation of the silencer. Such a derepression also occurs in other neuronal lineages. In nonneuronal lineages, SCG10 remains in a repressed state. Later in sympathoadrenal development, the gene is either further induced by FGF and NGF in sympathetic neurons or suppressed by $\mathrm{GC}$ in adrenal chromaffin cells. This induction or suppression is superimposed on the derepressed state of the gene. Note that cells indicated in white letters are those in which the silencer is active; those indicated in black are cells in which the silencer is inactive. (Reprinted, with permission, from Mori et al. 1990.) 
from a repression imposed by sequence-specific silencer-binding factors. Whatever the case, we imagine that such a derepression is a prerequisite for the subsequent modulation (Stein et al. 1988a) of SCG10 expression by FGF/NGF and GC. It is likely, however, that the acquisition of such a derepressed state is insufficient to confer full regulatability on these genes. Progenitor cells must also acquire receptors for FGF and NGF, as well as signal-transduction components and transcription factors that specifically interact with the SCG10 gene in its derepressed state. Nevertheless, we believe that the derepression of SCG10 may define an important developmental step, in which an apparently cell-intrinsic mechanism affects the genomic response of a progenitor cell to a set of specific environmental influences. An understanding of the mechanism of SCG10 derepression may therefore yield insights into the molecular basis for the segregation of neural crest cells into the sympathoadrenal and other neuronal sublineages.

\section{ACKNOWLEDGMENTS}

Some of the work described in this paper was supported by National Institutes of Health (NIH) grant NS-23476, Searle Scholars and National Science Foundation Presidential Young Awards to D.J.A., and a U.S.-Israeli Binational Science Foundation grant to R.S. We thank LiChing Lo and Steven Padilla for technical assistance and Helen Walsh for preparation of the manuscript. We are also grateful to our colleagues Paul Patterson and Barbara Wold for frequent helpful discussions. S.J.B. was supported by a Damon Runyon-Walter Winchell postdoctoral fellowship and D.J.V. and C.W.W. by NIH fellowships.

\section{REFERENCES}

Anderson, D.J. 1988. Cell fate and gene expression in the developing neural crest. NATO ASI Ser. H 22: 188.

—_ 1989. The neural crest cell lineage problem: Neuropoiesis? Neuron 3: 1

Anderson, D.J. and R. Axel. 1985. Molecular probes for the development and plasticity of neural crest derivatives. Cell 42: 649 .

Anderson, D.J. and R. Axel. 1986. A bipotential neuroendocrine precursor whose choice of cell fate is determined by NGF and glucocorticoid. Cell 47: 1079 .

Baroffio, A., E. Dupin, and N.M. Le Douarin. 1988. Cloneforming ability and differentiation potential of migratory neural crest cells. Proc. Natl. Acad. Sci. 85: 5325.

Birren, S.J. and D.J. Anderson. 1990. A v-myc-immortalized sympathoadrenal progenitor cell line in which neuronal differentiation is initiated by FGF but not NGF. Neuron 4: 189.

Bronner-Fraser, M. and S. Fraser. 1989. Developmental potential of avian trunk neural crest cells in situ. Neuron 3: 755.

Burch, J.B.E. and H. Weintraub. 1983. Temporal order of chromatin structure changes associated with activation of the major chicken vitellogenin gene. Cell 33: 65.

Chandler, V.L., B.A. Maler, and K.R. Yamomoto. 1983. DNA sequences bound specifically by glucocorticoid re- ceptor in vitro render a heterologous promoter hormone responsive in vivo. Cell 33: 489.

Claude, P.. I.M. Parada, K.A. Gordon, P.A. D'Amore, and J.A. Wagner. 1988. Acidic fibroblast growth factor stimulates adrenal chromaffin cells to proliferate and to extend neurites, but is not a long-term survival factor. Neuron 1: 783

Cochard, P., M. Goldstein, and I. Black. 1979. Initial development of the noradrenergic phenotype in automatic neuroblasts of the rat embryo in vivo. Dev. Biol. 71: 100.

Coughlin. M.D. and M.D. Collins. 1985. Nerve growth factorindependent development of embryonic mouse sympathetic neurons in dissociated cell culture. Dev. Biol. 110: 392 .

Davies, A.M. C. Bandtlow, R. Heumann, S. Korsching, H. Rohrer, and H. Thoenen. 1987. The site and timing of nerve growth factor (NGF) synthesis in developing skin in relation to its innervation by sensory neurons and their expression of NGF receptors. Nature 326: 353.

Doherty, P., P. Seaton, T. Flanigan, and F.S. Walsh. 1988. Factors controlling the expression of the NGF receptor in PC12 cells. Neurosci. Letl. 92: 222.

Doupe, A.J., S.C. Landis, and P.H. Patterson. 1985a. Environmental influences in the development of neural crest derivatives: Glucocorticoids, growth factors and chromaffin cell plasticity. J. Neurosci. 5: 2119.

Doupe. A.J., P.H. Patterson, and S.C. Landis. 1985b. Small intensely fluorescent (SIF) celis in culture: Role of glucocorticoids and growth factors in their development and phenotypic interconversions with other neural crest derivátives. J. Neurosci. 5: 2143.

Ernsberger, U., D. Edgar, and H. Rohner. 1989. The survival of early chick sympathetic neurons in vitro is dependent on a suitable substrate but independent of NGF. Dev. Biol. 135: 250

Greene, L.A. and A.S. Tischler. 1976. Establishment of a noradrenergic clonal line of rat adrenal phaeochromocytoma cells which respond to nerve growth factor. Proc. Natl. Acad. Sci. 73: 2424.

Gross, D.S. and W.T. Garrard. 1988. Nuclear hypersensitive sites in chromatin. Annu. Rev. Biochem. 57: 159.

Hébert, J.M., C. Basilico, M. Goldfarb, O. Haub, and G.R. Martin. 1990. Isolation of cDNAs encoding four mouse FGF family members and characterization of their expression patterns during embryogenesis. Dev. Biol. (in press).

Holt. C.E., T.W. Bertsch, H.M. Ellis, and W.A. Harris. 1988. Cellular determination in the Xenopus retina is independent of lineage and birth date. Neuron 1: 15.

Kalcheim, C. and G. Neufeld. 1990. Expression of basic fibroblast growth factor in the nervous system of early avian embryos. Development 109: 203.

Korsching. S. and H. Thoenen. 1988. Developmental changes of nerve growth factor levels in sympathetic ganglia and their target organs. Dev. Biol. 126: 40 .

Leonard, D.G.B., E.B. Ziff, and L.A. Greene. 1987. Identification and characterization of mRNAs regulated by nerve growth factor in PC12 cells. Mol. Cell. Biol. 7: 3156 .

McKnight, S.L. and R. Kingsbury. 1982. Transcriptional control signals of a eukaryotic protein-coding gene. Science 217: 316

Mori, N., R. Stein, O. Sigmund, and D.J. Anderson. 1990. A cell type-preferred silencer element that controls the neural-specific expression of the SCG10 gene. Neuron 4: 583 .

Raff, M. C. 1989. Glial cell diversification in the rat optic nerve. Science 243: 1450.

Robins, D.M. I. Paek, P.H. Seeburg, and R. Axel. 1982. Regulated expression of human growth hormone genes in mouse cells. Cell 29: 623 .

Seidl, K. and K. Unsicker. 1989. The determination of the adrenal medullary cell fate during embryogenesis. Dev. Biol. 136: 481

Sheng. M. and M.E. Greenberg. 1990. The regulation and 
function of c-fos and other immediate early genes in the nervous system. Neuron 4: 477.

Sieber-Blum, M. and A.M. Cohen. 1980. Clonal analysis of quail neural crest cells: They are pluripotent and differentiate in vitro in the absence of non crest cells. Dev. Biol. 80: 96.

Stein, R. O. Sigmund, and D.J. Anderson. 1988a. The induction of a neural-specific gene, SCG10, by nerve growth factor in PC12 cells is transcriptional, protein synthesis dependent, and glucocorticoid inhibitable. Dev. Biol. 127: 316.

Stein, R., N. Mori, K. Matthews, L.-C. Lo, and D.J. Anderson. 1988. The NGF-inducible SCG10 mRNA encodes a novel membrane-bound protein present in growth cones and abundant in developing neurons. Neuron 1: 463.

Stemple, D.L., N.K. Mahanthappa. and D.J. Anderson. 1988. Basic FGF induces neuronal differentiation, cell division, and NGF dependence in chromaffin cells: A sequence of events in sympathetic development. Neuron 1: 517 .
Turner, D.H. and C. Cepko. 1987. A common progenitor for neurons and glia persists in rat retina late in development. Nature 328: 131.

Unsicker, K., B. Drisch, J. Otten, and H. Thoenen. 1978. Nerve growth factor-induced fiber outgrowth from isolated rat adrenal chromaffin cells: Impairment by glucocorticoids. Proc. Natl. Acad. Sci. 75: 3498

Vandenbergh, D.J.. C.W. Wuenschell, N. Mori, and D.J. Anderson. 1989. Chromatin structure as a molecular marker of cell lineage and developmental potential in neural crest-derived chromaffin cells. Neuron 3:507.

Wetts, R. and S.E. Fraser. 1988. Multipotent precursors can give rise to all major cell types of the frog retina. Science 239: 1142 .

Wuenschell, C.W., N. Mori, and D.J. Anderson. 1990. Analysis of SCG10 gene expression in transgenic mice reveals that neural specificity is achieved through selective derepression. Neuron 4: 595 